\title{
Optimal decay rates for the compressible viscoelastic flows
}

\author{
Yin Li, ${ }^{1,2,3, a)}$ Ruiying Wei, ${ }^{1,2, b)}$ and Zheng-an Yao ${ }^{2, c)}$ \\ ${ }^{1}$ School of Mathematics and Statistics, Shaoguan University, 512005 Shaoguan, \\ People's Republic of China \\ ${ }^{2}$ Department of Mathematics, Sun Yat-sen University, 510275 Guangzhou, \\ People's Republic of China \\ ${ }^{3}$ School of Mathematics and Statistics, Yunnan University, 650091 Kunming, \\ People's Republic of China
}

(Received 15 May 2015; accepted 7 November 2016; published online 29 November 2016)

In this paper, we study the compressible viscoelastic flows in three-dimensional whole space. Under the assumption of small initial data, we establish the unique global solution by the energy method. Furthermore, we obtain the time decay rates of the higher-order spatial derivatives of the solution if the initial data belong to $L^{1}\left(\mathbb{R}^{3}\right)$ additionally. Published by AIP Publishing. [http://dx.doi.org/10.1063/1.4967975]

\section{INTRODUCTION}

In this paper, we consider the compressible viscoelastic flows

$$
\left\{\begin{array}{l}
\rho_{t}+\operatorname{div}(\rho u)=0, \\
(\rho u)_{t}+\operatorname{div}(\rho u \otimes u)-\mu \Delta u-(\mu+\lambda) \nabla \operatorname{div} u+\nabla p(\rho)=\alpha \operatorname{div}\left(\rho F F^{\top}\right), \\
F_{t}+u \cdot \nabla F=\nabla u F .
\end{array}\right.
$$

The equations are considered in $\mathbb{R}^{3} \times[0,+\infty)$, where $\rho, u \in \mathbb{R}^{3}, F \in M^{3 \times 3}$ (the set of $3 \times 3$ matrices with positive determinants) represent the density, the velocity, and the deformation gradient, respectively. The pressure $P=P(\rho)$ is a smooth function of $\rho$ for $\rho>0, F^{\top}$ means the transpose matrix of $F$. $\mu$ and $\lambda$ denote the shear viscosity and the bulk viscosity coefficients of the flow satisfying

$$
\mu>0,2 \mu+3 \lambda \geq 0 \text {. }
$$

The positive parameter $\alpha$ represents the speed of propagation of shear waves. For system (1.1), the corresponding elastic energy is chosen to be the special form of the Hookean linear elasticity

$$
W(F)=\frac{\alpha}{2}|F|^{2}+\frac{1}{\rho} \int_{0}^{\rho} P(s) d s, \alpha>0,
$$

which, however, does not reduce the essential difficulties for analysis.

We investigate the Cauchy problem of system (1.1) with the following initial condition:

$$
\left.(\rho, u, F)\right|_{t=0}=\left(\rho_{0}(x), u_{0}(x), F_{0}(x)\right), x \in \mathbb{R}^{3} .
$$

We also assume that

$$
\operatorname{div}\left(\rho F^{\top}\right)=0, F^{l k}(0) \nabla_{l} F^{i j}(0)=F^{l j}(0) \nabla_{l} F^{i k}(0) .
$$

It is standard that the condition (1.2) is preserved by the flow, which has been proved in Refs. 8 and 20 .

For the incompressible viscoelastic fluids, there has been much important progress on classical solutions; refer to Refs. 1, 16, and 24 and references therein. However, the global existence of

\footnotetext{
a) Electronic address: liyin2009521@163.com.

b) Author to whom correspondence should be addressed. Electronic mail: weiruiying521@163.com.

c) Electronic address: mcsyao@mail.sysu.edu.cn.
} 
weak solutions to the incompressible viscoelastic flows with large initial data is an outstanding open problem, although there has been some progress in this direction. ${ }^{15,17,18}$ For the compressible viscoelastic flows, the local existence of a multidimensional strong solution was obtained in Ref. 7 , and the global existence of a strong solution with the lowest regularity was shown in Refs. 8 and 20.

The existence and convergence rate of the solutions is an important problem in the PDE theory. The decay rate of solutions to the Navier-Stokes system has been investigated extensively, see Refs. 2-6, 13, 14, 19, 22, 23, and 25 and references therein. However, due to the lack of dissipation on the deformation tensor $\mathrm{F}$, it seems that the approaches employed in the previously mentioned works (e.g., the Fourier splitting method ${ }^{22,23}$ ) fail to apply, so far, there are few results on the large time behavior, especially about the higher-order spatial derivatives of the solution. Recently, $\mathrm{Hu}-\mathrm{Wu}^{9}$ proved the global existence of the strong solutions by the standard energy method under the condition that the initial data are close to the constant equilibrium state in $\mathrm{H}^{2}$-framework. And if additionally the initial data belong to $L^{1}$, the optimal convergence rates of the solutions in $L^{p}$-norm with $2 \leq p \leq 6$ and optimal convergence rates of their spatial derivatives in $L^{2}$-norm are obtained as follows:

$$
\begin{gathered}
\|(\varrho, v, E)(t)\|_{L^{p}} \leq C(1+t)^{-\frac{3}{2}\left(1-\frac{1}{p}\right)}, \quad \forall p \in[2,6], \\
\|\nabla(\varrho, v, E)(t)\|_{H^{1}} \leq C(1+t)^{-\frac{5}{4}} .
\end{gathered}
$$

In Ref. 10, by introducing a new decomposition via Helmholtz's projections, $\mathrm{Hu}-\mathrm{Wu}$ first provide an alternative proof on the existence of global smooth solutions near equilibrium, then they obtained the optimal $L^{2}$ decay rates for the global smooth solutions and their spatial derivatives for incompressible viscoelastic fluids in the whole space $\mathbb{R}^{d}(d=2,3)$ with additional assumptions that the initial data belong to $L^{1}$ and their Fourier modes do not degenerate at low frequencies. In Ref. 12, the authors established the local and global well posedness in the $L^{p}$ based critical Besov space and give a time decay rate in the Besov space framework. And in Ref. 11, the authors got the optimal time decay rate when the initial data were just small in the critical Besov space framework.

In this paper, we first establish the global solution by the energy method (1.1) under the assumption that the $H^{3}$ norm of the initial date is small, but the higher order derivatives can be arbitrarily large. Then we establish the time decay rates for the compressible viscoelastic flows by energy estimates and the Fourier splitting method by assuming that the initial data belongs to $L^{1}\left(\mathbb{R}^{3}\right)$ additionally.

Notation Throughout this paper, $\nabla^{l}$ with an integer $l \geq 0$ stands for the usual any spatial derivatives of order $l$. When $l<0$ or $l$ is not a positive integer, $\nabla^{l}$ stands for $\Lambda^{l}$ defined by $\Lambda^{s} u=$ $\mathscr{F}^{-1}\left(|\xi|^{s} \hat{u}(\xi)\right)$, where $\hat{u}$ is the Fourier transform of $u$ and $\mathscr{F}^{-1}$ its inverse. We will employ the notation $A \lesssim B$ to mean that $A \leq C B$ for a universal constant $C>0$ that only depends on the parameters coming from the problem. For the sake of conciseness, we write $\|(A, B)\|_{X}:=\|A\|_{X}+\|B\|_{X}$.

In this subsection, we first reformulate the system (1.1). Without loss of generality, we assume $P^{\prime}(1)>0$, and denote $\chi_{0}=\left(P^{\prime}(1)\right)^{-\frac{1}{2}}$. For $\rho>0$, system $(1.1)$ can be rewritten as

$$
\left\{\begin{array}{l}
\rho_{t}+\rho \operatorname{div} u+u \nabla \rho=0, \\
u_{t}^{i}+u \cdot \nabla u^{i}-\frac{1}{\rho}\left(\mu \Delta u^{i}+(\mu+\lambda) \nabla_{i} \operatorname{div} u\right)+\frac{P^{\prime}(\rho)}{\rho} \nabla_{i} \rho=\alpha F^{j k} \nabla_{j} F^{i k}, \\
F_{t}+u \cdot \nabla F=\nabla u F,
\end{array}\right.
$$

where we used the condition $\operatorname{div}\left(\rho F^{\top}\right)=0$ for all $t \geq 0$, which ensures that the $i$ th component of the vector $\operatorname{div}\left(\rho F F^{\top}\right)=0$ is

$$
\nabla_{j}\left(\rho F^{i k} F^{j k}\right)=\rho F^{j k} \nabla_{j} F^{i k}+F^{i k} \nabla_{j}\left(\rho F^{j k}\right)=\rho F^{j k} \nabla_{j} F^{i k} .
$$

We denote

$$
\varrho(t, x)=\rho\left(\chi_{0}^{2} t, \chi_{0} x\right)-1, v(t, x)=\chi_{0} u\left(\chi_{0}^{2} t, \chi_{0} x\right), E(t, x)=F\left(\chi_{0}^{2} t, \chi_{0} x\right)-I,
$$


then

$$
\left\{\begin{array}{l}
\varrho_{t}+\operatorname{div} v=-\varrho \operatorname{div} v-v \cdot \nabla \varrho \\
v_{t}^{i}-\mu \Delta v^{i}-(\mu+\lambda) \nabla_{i} \operatorname{div} v+\nabla_{i} \varrho-a \nabla_{j} E^{i j}=f \\
E_{t}-\nabla v=\nabla v E-v \cdot \nabla E
\end{array}\right.
$$

where

$$
f^{i}=a E^{j k} \nabla_{j} E^{i k}-g(\varrho)\left(\mu \Delta v^{i}+(\mu+\lambda) \nabla_{i} \operatorname{div} v\right)-v \cdot \nabla v^{i}-h(\varrho) \nabla_{i} \varrho,
$$

and the nonlinear functions of $\varrho$ are defined by

$$
g(\varrho)=\frac{\varrho}{\varrho+1}, \quad h(\varrho)=\frac{P^{\prime}(\varrho+1)}{(\varrho+1) P^{\prime}(1)}-1 .
$$

Without loss of generality, we will assume that $a=1$ for the rest of this paper.

Our main results are stated in the following theorem.

Theorem 1.1. Let $N \geq 3$, assume that $\left(\varrho_{0}, v_{0}, E_{0}\right) \in H^{N}\left(\mathbb{R}^{3}\right)$. Then there exists a constant $\delta_{0}>$ 0 such that if

$$
\left\|\left(\varrho_{0}, v_{0}, E_{0}\right)\right\|_{H^{3}} \leq \delta_{0},
$$

then the problem (1.1) has a unique global solution $(\varrho(t), v(t), E(t))$ satisfying that for all $t \geq 0$,

$$
\begin{aligned}
& \|(\varrho, v, E)(t)\|_{H^{N}}^{2}+\int_{0}^{t}\left(\|\nabla \varrho(\tau)\|_{H^{N-1}}^{2}+\|\nabla v(\tau)\|_{H^{N}}^{2}+\|\nabla E(\tau)\|_{H^{N-1}}^{2}\right) d \tau \\
\leq & C\left\|\left(\varrho_{0}, v_{0}, E_{0}\right)\right\|_{H^{N}}^{2} .
\end{aligned}
$$

If further, $\left(\varrho_{0}, v_{0}, E_{0}\right) \in L^{1}\left(\mathbb{R}^{3}\right)$, then

$$
\left\|\nabla^{l}(\varrho, v, E)(t)\right\|_{H^{N-l}} \leq C(1+t)^{-\frac{3+2 l}{4}}, \text { for } l=0,1, \cdots, N-1,
$$

and for $2 \leq p \leq \infty$, there holds

$$
\left\|\nabla^{l}(\varrho, v, E)(t)\right\|_{L^{p}} \leq C(1+t)^{-\frac{l}{2}-\frac{3}{2}\left(1-\frac{1}{p}\right)},
$$

especially,

$$
\left\|\nabla^{l}(\varrho, v, E)(t)\right\|_{L^{\infty}} \leq C(1+t)^{-\frac{3+l}{2}} .
$$

\section{ENERGY ESTIMATES}

In this subsection, we will derive the a priori nonlinear energy estimates for the system (1.5). Hence we assume a priori that for sufficiently small $\delta>0$,

$$
\sqrt{\mathcal{E}_{0}^{3}(t)}=\|(\varrho, v, E)(t)\|_{H^{3}} \leq \delta .
$$

First of all, by (2.1) and Sobolev's inequality, we obtain

$$
\frac{1}{2} \leq \varrho+1 \leq \frac{3}{2} .
$$

Hence, we immediately have

$$
|g(\varrho)|,|h(\varrho)| \leq C|\varrho|,\left|g^{(k)}(\varrho)\right|,\left|h^{(k)}(\varrho)\right| \leq C \text { for any } k \geq 1,
$$

where $g, h$ are nonlinear functions of $\varrho$ defined by (1.8)

We will establish the global existence of solution for the compressible viscoelastic flows. For this purpose, we begin with the energy estimates including $\varrho, v$ and $E$ themselves, the following lemma may refer to Ref. 26, and we state the results here for the sake of convenience. 
Lemma 2.1. If $\sqrt{\mathcal{E}_{0}^{3}(t)} \leq \delta$, then for $k=0, \cdots, N-1$, we have

$$
\begin{gathered}
\frac{d}{d t} \int_{\mathbb{R}^{3}}\left(\left|\nabla^{k} \varrho\right|^{2}+\left|\nabla^{k} v\right|^{2}+\left|\nabla^{k} E\right|^{2}\right) d x+C\left\|\nabla^{k+1} v\right\|_{L^{2}}^{2} \lesssim \delta\left\|\nabla^{k+1}(\varrho, v, E)\right\|_{L^{2}}^{2}, \\
\frac{d}{d t} \int_{\mathbb{R}^{3}}\left(\left|\nabla^{k+1} \varrho\right|^{2}+\left|\nabla^{k+1} v\right|^{2}+\left|\nabla^{k+1} E\right|^{2}\right) d x+C\left\|\nabla^{k+2} v\right\|_{L^{2}}^{2} \\
\lesssim \delta\left(\left\|\nabla^{k+1} \varrho\right\|_{L^{2}}^{2}+\left\|\nabla^{k+2} v\right\|_{L^{2}}^{2}+\left\|\nabla^{k+1} E\right\|_{L^{2}}^{2}\right), \\
\frac{d}{d t} \int_{\mathbb{R}^{3}} \nabla^{k} v \cdot \nabla^{k+1} \varrho d x+C\left\|\nabla^{k+1} \varrho\right\|_{L^{2}}^{2} \lesssim\left\|\nabla^{k+1} v\right\|_{L^{2}}^{2}+\left\|\nabla^{k+2} v\right\|_{L^{2}}^{2}+\left\|\nabla^{k+1} E\right\|_{L^{2}}^{2}, \\
\frac{d}{d t} \int_{\mathbb{R}^{3}} \nabla^{k+1} v \cdot \nabla^{k} E d x+\left\|\nabla^{k+1} E\right\|_{L^{2}}^{2} \lesssim\left\|\nabla^{k+1} \varrho\right\|_{L^{2}}^{2}+\left\|\nabla^{k+1} v\right\|_{L^{2}}^{2}+\left\|\nabla^{k+2} v\right\|_{L^{2}}^{2} .
\end{gathered}
$$
1.1 .

Next, we will combine all the energy estimates that we have derived to prove (1.10) of Theorem

Proof. We first close the energy estimates at each $l$ th level in our weaker sense. Let $N \geq 3$ and $0 \leq l \leq m-1$ with $1 \leq m \leq N$. Summing up the estimates (2.4) of Lemma 2.1 from $k=l$ to $m-1$, since $\sqrt{\mathcal{E}_{0}^{3}} \leq \delta$ is small, we obtain

$$
\frac{d}{d t} \sum_{l \leq k \leq m-1}\left\|\nabla^{k}(\varrho, v, E)\right\|_{L^{2}}^{2}+C \sum_{l+1 \leq k \leq m}\left\|\nabla^{k} v\right\|_{L^{2}}^{2} \lesssim \delta \sum_{l+1 \leq k \leq m}\left\|\nabla^{k}(\varrho, v, E)\right\|_{L^{2}}^{2} .
$$

Let $k=m-1$ in the estimates (2.5) of Lemma 2.1, we have

$$
\frac{d}{d t}\left\|\nabla^{m}(\varrho, v, E)\right\|_{L^{2}}^{2}+C\left\|\nabla^{m+1} v\right\|_{L^{2}}^{2} \lesssim \delta\left(\left\|\nabla^{m} \varrho\right\|_{L^{2}}^{2}+\left\|\nabla^{m+1} v\right\|_{L^{2}}^{2}+\left\|\nabla^{m} E\right\|_{L^{2}}^{2}\right) .
$$

Adding the inequality (2.9) to (2.8), we get

$$
\frac{d}{d t} \sum_{l \leq k \leq m}\left\|\nabla^{k}(\varrho, v, E)\right\|_{L^{2}}^{2}+C_{1} \sum_{l+1 \leq k \leq m+1}\left\|\nabla^{k} v\right\|_{L^{2}}^{2} \leq C_{2} \delta \sum_{l+1 \leq k \leq m}\left\|\nabla^{k}(\varrho, E)\right\|_{L^{2}}^{2} .
$$

Summing up the estimates (2.6), (2.7) of Lemma 2.1 from $k=l$ to $m-1$, respectively, and combining the results, we have

$$
\begin{aligned}
& \frac{d}{d t} \sum_{l \leq k \leq m-1} \int_{\mathbb{R}^{3}}\left(\nabla^{k} v \cdot \nabla^{k+1} \varrho+\nabla^{k+1} v \cdot \nabla^{k} E\right) d x+C_{3} \sum_{l+1 \leq k \leq m}\left\|\nabla^{k}(\varrho, E)\right\|_{L^{2}}^{2} \\
\leq & C_{4}\left(\sum_{l+1 \leq k \leq m}\left\|\nabla^{k}(\varrho, E)\right\|_{L^{2}}^{2}+\sum_{l+1 \leq k \leq m+1}\left\|\nabla^{k+1} v\right\|_{L^{2}}^{2}\right) .
\end{aligned}
$$

Multiplying (2.11) by $\frac{2 C_{2} \delta}{C_{3}}$, adding it to (2.10), since $\delta>0$ is small, we deduce that there exists a constant $C_{5}>0$ such that for $0 \leq l \leq m-1$

$$
\begin{aligned}
& \frac{d}{d t}\left\{\sum_{l \leq k \leq m}\left\|\nabla^{k}(\varrho, v, E)\right\|_{L^{2}}^{2}+\frac{2 C_{2} \delta}{C_{3}} \sum_{l \leq k \leq m-1} \int_{\mathbb{R}^{3}}\left(\nabla^{k} v \cdot \nabla^{k+1} \varrho+\nabla^{k+1} v \cdot \nabla^{k} E\right) d x\right\} \\
& \quad+C_{5}\left\{\sum_{l+1 \leq k \leq m}\left\|\nabla^{k}(\varrho, E)\right\|_{L^{2}}^{2}+\sum_{l+1 \leq k \leq m+1}\left\|\nabla^{k} v\right\|_{L^{2}}^{2}\right\} \leq 0 .
\end{aligned}
$$

Next, we define $\mathcal{E}_{l}^{m}(t)$ to be $C_{5}^{-1}$ times the expression under the time derivative in (2.12). Observe that since $\delta$ is small, $\mathcal{E}_{l}^{m}(t)$ is equivalent to $\left\|\nabla^{l}(\varrho, v, E)(t)\right\|_{H^{m-l}}^{2}$, that is, there exists a constant $C_{6}>0$ such that for $0 \leq l \leq m-1$

$$
C_{6}^{-1}\left\|\nabla^{l}(\varrho, v, E)(t)\right\|_{H^{m-l}}^{2} \leq \mathcal{E}_{l}^{m}(t) \leq C_{6}\left\|\nabla^{l}(\varrho, v, E)(t)\right\|_{H^{m-l}}^{2} .
$$


Then we may write (2.12) as that for $0 \leq l \leq m-1$

$$
\frac{d}{d t} \mathcal{E}_{l}^{m}(t)+\left\|\nabla^{l+1} \varrho\right\|_{H^{m-l-1}}^{2}+\left\|\nabla^{l+1} v\right\|_{H^{m-l}}^{2}+\left\|\nabla^{l+1} E\right\|_{H^{m-l-1}}^{2} \leq 0 .
$$

Taking $l=0$ and $m=3$ in (2.14), and then integrating directly in time, we get

$$
\|(\varrho, v, E)(t)\|_{H^{3}}^{2} \lesssim \mathcal{E}_{0}^{3}(t) \lesssim \mathcal{E}_{0}^{3}(0) \lesssim\left\|\left(\varrho_{0}, v_{0}, E_{0}\right)\right\|_{H^{3}}^{2} .
$$

By a standard continuity argument, this closes the a priori estimates (2.1) if we assume that $\left\|\left(\varrho_{0}, v_{0}, E_{0}\right)\right\|_{H^{3}}^{2} \leq \delta_{0}$ is sufficiently small. This in turn allows us to take $l=0$ and $m=N$ in (2.14), and then integrate it directly in time to obtain

$$
\|(\varrho, v, E)(t)\|_{H^{N}}^{2}+\int_{0}^{t}\|\nabla \varrho(\tau)\|_{H^{N-1}}^{2}+\|\nabla v(\tau)\|_{H^{N}}^{2}+\|\nabla E(\tau)\|_{H^{N-1}}^{2} d \tau \leq C\left\|\left(\varrho_{0}, v_{0}, E_{0}\right)\right\|_{H^{N}}^{2} .
$$

This proved (1.10).

\section{CONVERGENCE RATE OF THE SOLUTION}

In this section, we shall prove the decay rates of the solution stated in Theorem 1.1 under additional assumptions that the initial data belong to $L^{1}$. In Ref. 9, the authors proved the following lemma, which utilizes the decay-in-time estimates for the linearized system to control the first order derivatives by the higher order derivatives.

Lemma 3.1. Let $(\varrho, v, E)$ be the solution to the initial value problem (1.6), under the assumption $\sqrt{\mathcal{E}_{0}^{2}(t)}=\|(\varrho, v, E)(t)\|_{H^{2}} \leq \delta$, then we have

$$
\|\nabla(\varrho, v, E)\|_{L^{2}} \leq C(1+t)^{-\frac{5}{4}}\left(K_{0}+\delta \sqrt{N(t)}\right),
$$

where $K_{0}=\left\|\left(\varrho_{0}, v_{0}, E_{0}\right)\right\|_{L^{1} \cap H^{2}}, \quad N(t)=\sup _{0 \leq \tau \leq t}(1+\tau)^{\frac{5}{2}}\|\nabla(\varrho, v, E)(\tau)\|_{H^{1}}^{2}$.

Now, we first establish the following time decay rates for the compressible viscoelastic flows (1.6).

Lemma 3.2. Under the assumptions of Theorem 1.1, the global solution $(\varrho, v, E)$ of problem (1.6) satisfies

$$
\left\|\nabla^{l}(\varrho, v, E)(t)\right\|_{H^{N-l}} \leq C(1+t)^{-\frac{3+2 l}{4}}, \text { for } l=0,1 .
$$

Proof. Adding $\left\|\nabla^{l}(\varrho, v, E)(t)\right\|_{L^{2}}^{2}$ to both sides of (2.14) gives

$$
\frac{d}{d t} \mathcal{E}_{l}^{m}(t)+C \mathcal{E}_{l}^{m}(t) \leq\left\|\nabla^{l}(\varrho, v, E)(t)\right\|_{L^{2}}^{2} .
$$

Taking $l=1$ and $m=N$ in (3.3), we get

$$
\frac{d}{d t} \mathcal{E}_{1}^{N}(t)+C \mathcal{E}_{1}^{N}(t) \leq\|\nabla(\varrho, v, E)(t)\|_{L^{2}}^{2} .
$$

It follows from the Gronwall inequality and Lemma 3.1 that

$$
\begin{aligned}
\mathcal{E}_{1}^{N}(t) & \leq \mathcal{E}_{1}^{N}(0) e^{-C t}+\int_{0}^{t} e^{-C(t-\tau)}\|\nabla(\varrho, v, E)(t)\|_{L^{2}}^{2} d \tau \\
& \leq \mathcal{E}_{1}^{N}(0) e^{-C t}+C \int_{0}^{t} e^{-C(t-\tau)}\left(K_{0}^{2}+\delta^{2} N(\tau)\right)(1+\tau)^{-\frac{5}{2}} d \tau \\
& \leq \mathcal{E}_{1}^{N}(0) e^{-C t}+C\left(K_{0}^{2}+\delta^{2} M(t)\right) \int_{0}^{t} e^{-C(t-\tau)}(1+\tau)^{-\frac{5}{2}} d \tau \\
& \leq \mathcal{E}_{1}^{N}(0) e^{-C t}+C\left(K_{0}^{2}+\delta^{2} M(t)\right)(1+t)^{-\frac{5}{2}} \\
& \leq C\left(K_{0}^{2}+\delta^{2} M(t)\right)(1+t)^{-\frac{5}{2}},
\end{aligned}
$$


where $M(t)=\sup _{0 \leq \tau \leq t}(1+t)^{\frac{5}{2}} \mathcal{E}_{1}^{N}(t)$ and we have used the fact

$$
\begin{aligned}
& \int_{0}^{t} e^{-C(t-\tau)}(1+\tau)^{-\frac{5}{2}} d \tau \\
= & \int_{0}^{\frac{t}{2}} e^{-C(t-\tau)}(1+\tau)^{-\frac{5}{2}} d \tau+\int_{\frac{t}{2}}^{t} e^{-C(t-\tau)}(1+\tau)^{-\frac{5}{2}} d \tau \\
\leq & e^{-\frac{C t}{2}} \int_{0}^{\frac{t}{2}}(1+\tau)^{-\frac{5}{2}} d \tau+\left(1+\frac{t}{2}\right)^{-\frac{5}{2}} \int_{\frac{t}{2}}^{t} e^{-C(t-\tau)} d \tau \\
\leq & C(1+t)^{-\frac{5}{2}}
\end{aligned}
$$

Based on the definition of $M(t)$ and (3.5), we get

$$
M(t) \leq C\left(K_{0}^{2}+\delta^{2} M(t)\right)
$$

which implies

$$
M(t) \leq C,
$$

since $\delta>0$ is sufficiently small.

Hence, we have the following decay rates

$$
\|\nabla(\varrho, v, E)(t)\|_{H^{N-l}} \leq C(1+t)^{-\frac{5}{4}}
$$

which together with (1.3) implies (3.2).

Lemma 3.3. Under the assumptions of Theorem 1.1, the global solution $(\varrho, v, E)$ of problem (1.6) satisfies

$$
\left\|\nabla^{l}(\varrho, v, E)(t)\right\|_{H^{N-l}} \leq C(1+t)^{-\frac{3+2 l}{4}}, \text { for } l=0,1, \cdots, N-1 .
$$

Proof. We are ready to prove (3.8) by induction. When $l=0,1$, the inequality (3.8) has been established in Lemma 3.2. Suppose (3.8) holds for the case $l=k-1$, and $k=2,3, \cdots, N-1$, that is

$$
\left\|\nabla^{k-1}(\varrho, v, E)(t)\right\|_{H^{N-k+1}} \leq C(1+t)^{-\frac{1+2 k}{4}} .
$$

We need to show (3.8) holds for $l=k$. Let $l=k$ and $m=N$ in the estimates (2.14), we have

$$
\frac{d}{d t} \mathcal{E}_{k}^{N}(t)+\left\|\nabla^{k+1} \varrho\right\|_{H^{N-k-1}}^{2}+\left\|\nabla^{k+1} v\right\|_{H^{N-k}}^{2}+\left\|\nabla^{k+1} E\right\|_{H^{N-k-1}}^{2} \leq 0 .
$$

Adding $\left\|\nabla^{k+1}(\varrho, E)(t)\right\|_{L^{2}}^{2}$ to both sides of (3.10) gives

$$
\frac{d}{d t} \mathcal{E}_{k}^{N}(t)+C\left(\left\|\nabla^{k+1}(\varrho, E)(t)\right\|_{L^{2}}^{2}+\left\|\nabla^{k+1} \varrho\right\|_{H^{N-k-1}}^{2}+\left\|\nabla^{k+1} v\right\|_{H^{N-k}}^{2}+\left\|\nabla^{k+1} E\right\|_{H^{N-k-1}}^{2}\right) \leq 0 .
$$

As in Ref. 21, we define

$$
S(t)=\left\{\xi \in \mathbb{R}^{3}:|\xi| \leq\left(\frac{a}{1+t}\right)^{\frac{1}{2}}\right\},
$$

for a constant $a$ that will be specified below. Then

$$
\begin{aligned}
\left\|\nabla^{k+1} \varrho\right\|_{L^{2}}^{2} & =\int_{\mathbb{R}^{3}}|\xi|^{2(k+1)}|\varrho|^{2} d \xi \geq \int_{\mathbb{R}^{3} / S}|\xi|^{2(k+1)}|\varrho|^{2} d \xi \\
& \geq \frac{a}{1+t} \int_{\mathbb{R}^{3} / S}|\xi|^{2 k}|\varrho|^{2} d \xi \\
& \geq \frac{a}{1+t} \int_{\mathbb{R}^{3}}|\xi|^{2 k}|\varrho|^{2} d \xi-\frac{a^{2}}{(1+t)^{2}} \int_{S}|\xi|^{2(k-1)}|\hat{\varrho}|^{2} d \xi \\
& \geq \frac{a}{1+t} \int_{\mathbb{R}^{3}}|\xi|^{2 k}|\varrho|^{2} d \xi-\frac{a^{2}}{(1+t)^{2}} \int_{\mathbb{R}^{3}}|\xi|^{2(k-1)}|\hat{\varrho}|^{2} d \xi .
\end{aligned}
$$


Thus, we have

$$
\left\|\nabla^{k+1} \varrho\right\|_{L^{2}}^{2} \geq \frac{a}{1+t}\left\|\nabla^{k} \varrho\right\|_{L^{2}}^{2}-\frac{a^{2}}{(1+t)^{2}}\left\|\nabla^{k-1} \varrho\right\|_{L^{2}}^{2} .
$$

Similarly, one has

$$
\begin{gathered}
\left\|\nabla^{k+1} E\right\|_{L^{2}}^{2} \geq \frac{a}{1+t}\left\|\nabla^{k} E\right\|_{L^{2}}^{2}-\frac{a^{2}}{(1+t)^{2}}\left\|\nabla^{k-1} E\right\|_{L^{2}}^{2}, \\
\left\|\nabla^{k+1} v\right\|_{L^{2}}^{2} \geq \frac{a}{1+t}\left\|\nabla^{k} v\right\|_{L^{2}}^{2}-\frac{a^{2}}{(1+t)^{2}}\left\|\nabla^{k-1} v\right\|_{L^{2}}^{2} .
\end{gathered}
$$

Summing up the estimates (3.14) for $k$ from $k$ to $N$, one has

$$
\left\|\nabla^{k+1} v\right\|_{H^{N-k}}^{2} \geq \frac{a}{1+t}\left\|\nabla^{k} v\right\|_{H^{N-k}}^{2}-\frac{a^{2}}{(1+t)^{2}}\left\|\nabla^{k-1} v\right\|_{H^{N-k}}^{2} .
$$

Substituting the inequalities (3.12), (3.13), (3.15) into (3.11), and applying Lemma 3.2 and (3.9), we have

$$
\begin{aligned}
& \frac{d}{d t} \mathcal{E}_{k}^{N}(t)+\frac{C a}{1+t}\left(\left\|\nabla^{k}(\varrho, E)(t)\right\|_{L^{2}}^{2}+\left\|\nabla^{k+1}(\varrho, E)(t)\right\|_{H^{N-k-1}}^{2}+\left\|\nabla^{k+1} v\right\|_{H^{N-k}}^{2}\right) \\
\leq & \frac{C a^{2}}{(1+t)^{2}}\left(\left\|\nabla^{k-1}(\varrho, E)(t)\right\|_{L^{2}}^{2}+\left\|\nabla^{k-1} v\right\|_{H^{N-k}}^{2}\right) \\
\leq & C(1+t)^{-\frac{5+2 k}{2}},
\end{aligned}
$$

where we have used

$$
\frac{a}{1+t}\left\|\nabla^{k+1}(\varrho, E)(t)\right\|_{H^{N-k-1}}^{2} \leq\left\|\nabla^{k}(\varrho, E)(t)\right\|_{H^{N-k-1}}^{2},
$$

for some sufficiently large time $t \geq a-1$, such that $\frac{a}{1+t} \leq 1$.

This, together with the definition of $\mathcal{E}_{k}^{N}(t)$, implies that

$$
\frac{d}{d t} \mathcal{E}_{k}^{N}(t)+\frac{C a}{1+t} \mathcal{E}_{k}^{N}(t) \leq C(1+t)^{-\frac{5+2 k}{2}} .
$$

Choosing

$$
a=\frac{k+2}{C},
$$

and multiplying both sides of (3.16) by $(1+t)^{k+2}$, we get

$$
\frac{d}{d t}\left[(1+t)^{k+2} \mathcal{E}_{k}^{N}(t)\right] \leq C(1+t)^{-\frac{1}{2}} .
$$

Integrating (3.17) from 0 to $t$ yields

$$
\left\|\nabla^{k}(\varrho, v, E)(t)\right\|_{H^{N-k}}^{2} \leq C(1+t)^{-\frac{3+2 k}{2}} .
$$

Hence, we have verified that (3.8) holds on for the case $l=k$, this concludes the proof of lemma.

With Lemma 3.2 and Lemma 3.3 in hand, we are ready to prove Theorem 1.1:

Proof. With the help of Lemma 3.2 and Lemma 3.3, it is easy to obtain the conclusion (1.11). As for (1.12), by (1.11) and the Gagliardo-Nirenberg inequality,

$$
\left\|\nabla^{m} u\right\|_{L^{p}} \leq C\left\|\nabla^{m} u\right\|_{L^{2}}^{\theta}\|u\|_{L^{2}}^{1-\theta}, \frac{1}{p}-\frac{m}{3}=\left(\frac{1}{2}-\frac{m}{3}\right) \theta+\frac{1}{2}(1-\theta),
$$

the claim follows. 


\section{ACKNOWLEDGMENTS}

This work is partially supported by NNSFC (Grant Nos. 11271381, 11431015, and 11501373), China 973 Program (Grant No. 2011CB808002), Natural Science Foundation of Guangdong Province (Grant Nos. 2016A0300310019 and 2016A030307042), Guangdong Provincial culture of seedling of China (Grant No. 2013LYM0081), the Education research platform project of Guangdong Province (Grant No. 2014KQNCX208), the Education Reform Project of Guangdong Province (Grant No. 2015558).the Shaoguan Science and Technology Foundation (Grant Nos. 2014CX/K231 and 20157201), and Education Reform Project of Shaoguan University (Grant Nos. SYJY20121361 and SYJY20141576).

${ }^{1}$ J. Y. Chemin and N. Masmoudi, "About lifespan of regular solutions of equations related to viscoelastic fluids," SIAM J. Math. Anal. 33, 84-112 (2001).

${ }^{2}$ K. Deckelnick, "Decay estimates for the compressible Navier-Stokes equations in unbounded domains," Math. Z. 209, 115-130 (1992)

${ }^{3}$ K. Deckelnick, " $L^{2}$-decay for the compressible Navier-Stokes equations in unbounded domains," Commun. Partial Differ. Equations 18, 1445-1476 (1993).

${ }^{4}$ R. J. Duan, S. Ukai, T. Yang, and H. J. Zhao, "Optimal $L^{p}-L^{q}$ convergence rate for the compressible Navier-Stokes equations with potential force," J. Differ. Equations 238, 220-223 (2007).

${ }^{5}$ R. J. Duan, S. Ukai, T. Yang, and H. J. Zhao, "Optimal convergence rate for compressible Navier-Stokes equations with potential force," Math. Models Methods Appl. Sci. 17, 737-758 (2007).

${ }^{6}$ Y. Guo and Y. J. Wang, "Decay of dissipative equations and negative Sobolev spaces," Commun. Partial Differ. Equations 37, 2165-2208 (2012).

${ }^{7}$ X. P. Hu and D. H. Wang, "Local strong solution to the compressible viscoelastic flow with large data," J. Differ. Equations 249, 1179-1198 (2010).

${ }^{8}$ X. P. Hu and D. H. Wang, "Global existence for the multi-dimensional compressible viscoelastic flows," J. Differ. Equations 250, 1200-1231 (2011).

${ }^{9}$ X. P. Hu and G. C. Wu, "Global existence and optimal decay rates for three-dimensional compressible viscoelastic flows," SIAM J. Math. Anal. 45, 2815-2833 (2013).

${ }^{10} \mathrm{X}$. P. Hu and $\mathrm{H}$. Wu, "Long-time behavior and weak-strong uniqueness for incompressible viscoelastic flows," Discrete Contin. Dyn. Syst. 35, 3437-3461 (2015).

${ }^{11}$ J. X. Jia and J. G. Peng, "Optimal time decay rate for compressible viscoelastic equations in critical space," Appl. Anal. $1-21(2016)$

12 J. X. Jia, J. G. Peng, and Z. D. Mei, "Well-posedness and time-decay for compressible viscoelastic fluids in critical Besov space," J. Math. Anal. Appl. 418, 638-675 (2014).

${ }^{13}$ T. Kobayashi and Y. Shibata, "Decay estimates of solutions for the equations of motion of compressible viscous and heat-conductive gases in an exterior domain in $\mathbb{R}^{3}$," Commun. Math. Phys. 200, 621-659 (1999).

${ }^{14}$ T. Kobayashi and Y. Shibata, "Remark on the rate of decay of solutions to linearized compressible Navier-Stokes equations," Pac. J. Math. 207, 199-234 (2002).

${ }^{15}$ F. H. Lin, C. Liu, and P. Zhang, "On hydrodynamics of viscoelastic fluids," Commun. Pure Appl. Math. 58, 1437-1471 (2005).

${ }^{16}$ Z. Lei, C. Liu, and Y. Zhou, "Global existence for a 2D incompressible viscoelastic model with small strain," Commun Math. Sci. 5, 595-616 (2007).

${ }^{17}$ P. L. Lions and N. Masmoudi, "Global solutions for some Oldroyd models of non-Newtonian flows," Chin. Ann. Math., Ser. B 21, 131-146 (2000).

${ }^{18}$ T. P. Liu and W. K. Wang, "The pointwise estimates of diffusion waves for Navier-Stokes equations in odd multi-dimensions," Commun. Math. Phys. 196, 145-173 (1998).

${ }^{19}$ M. Oliver and E. S. Titi, "Remark on the rate of decay of higher order derivatives for solutions to the Navier-Stokes equations in $\mathbb{R}^{3}$," J. Funct. Anal. 172, 1-18 (2000).

${ }^{20}$ J. Z. Qian and Z. F. Zhang, "Global well-posedness for the compressible viscoelastic fluids near equilibrium," Arch. Ration. Mech. Anal. 198, 835-868 (2010).

${ }^{21}$ M. E. Schonbek, "On the decay of higher-order norms of the solutions of Navier-Stokes equations," Proc. R. Soc. Edinburgh: Sect. A Math. 126, 677-685 (1996).

${ }^{22}$ M. E. Schonbek, " $L^{2}$ decay of weak solutions of the Navier-Stokes equations," Arch. Ration. Mech. Anal. 88, 209-222 (1985).

${ }^{23}$ M. E. Schonbek, "Large time behaviour of solutions of the Navier-Stokes equations," Commun. Partial Differ. Equations 11, 733-763 (1986).

${ }^{24}$ T. Zhang and D. Y. Fang, "Global existence of strong solution for equations related to the incompressible viscoelastic fluids in the critical $L^{p}$ framework," SIAM J. Math. Anal. 44, 2266-2288 (2012).

${ }^{25}$ Y. J. Wang and Z. Tan, "Global existence and optimal decay rate for the strong solutions in $H^{2}$ to the compressible Navier-Stokes equations," Appl. Math. Lett. 24, 1778-1784 (2011).

${ }^{26}$ R. Y. Wei, Y. Li, and Z. A. Yao, "Decay of the compressible viscoelastic flows," Commun. Pure Appl. Anal. 15, 1603-1624 (2016). 